\title{
STUDY OF INSTRUMENTAL FACTORS AFFECTING X-RAY ISOCHROMAT SPECTRA
}

\author{
J.B. Peeka \\ Institute of Physics, Polish Academy of Sciences \\ Al. Lotników 32/46, 02-668 Warszawa, Poland
}

\begin{abstract}
In the present paper a model of an apparatus function for the bremsstrahlung isochromat spectroscopy method is proposed. The study presented in this work is based on experimental results obtained with a particular spectrometer working at the quantum energy of $\mathrm{Cr} K_{\alpha_{1}}(5414 \mathrm{eV})$. However, most of implications are general for the bremsstrahlung isochromat spectroscopy independently of particular construction of a spectrometer. From the theoretical considerations it was found that the total apparatus function, $F_{\mathrm{t}}$, is composed of two main subfunctions in such a manner that $F_{\mathrm{t}}$ is not necessarily constant along the isochromat spectrum. The properties of the function, as well as the question how it influences the measured spectra are discussed. Considerations presented in this paper are limited to the most essential instrumental factors broadening the bremsstrahlung isochromat spectra which can be described in terms of the apparatus function. In order to estimate the width of the apparatus function some experimental results of bremsstrahlung isochromat spectroscopy measurements of chosen substances with various apparatus settings are shown.
\end{abstract}

PACS numbers: $07.85 .+\mathrm{n}, 06.90 .+\mathrm{v}$

\section{Introduction}

The X-ray bremsstrahlung isochromat spectroscopy (BIS) is known to be a unique method of probing the density of unoccupied electron states (uDOS) independently of their symmetry types in the conducting and semiconducting solids. In the last years, the domain of the method has even been broadened with the studies of EXAFS-like (extended X-ray absorption fine structure) extended structure which appears in the spectra at higher excitation voltages. Due to a low yield of the isochromat spectra resulting in the well known low intensity of the BIS measurements, a good knowledge of the instrumental factors influencing the measurements is even more important than in other kinds of X-ray spectroscopy. It is particularly necessary when the BIS spectrometer uses quantum energies of few $\mathrm{keV}$ or higher. The differences in electron transition probabilities to the states of 
various symmetries depend on the isochromat energy. It is therefore often necessary to record the BIS spectra with the higher quantum energies in spite of their lower resolution comparing to low energetic BIS spectrometers. However, there is a lack of model which could adequately describe the instrumental factors affecting BIS measurements. It is also the case in the machine constructed and exploited in the Laboratory of X-ray Spectroscopy at the Institute of Physics. The isochromats of these higher energies are usually produced in a classical demountable X-ray tube with the measured sample placed on the anticathode (see Fig. 2). To obtain reasonable BIS intensities, rather high currents are applied in the tubes. It often results in significant broadening of the BIS spectra and in various effects caused by electron heating of the sample and the X-ray tube itself. It can be advantageous with such type of BIS spectrometer to study the influences of various factors on the apparatus function, because some of them are more pronounced.

The factors smearing or broadening the measured spectra are conventionally described in terms of apparatus (response) function. In the BIS method this broadening is caused by two groups of factors, the first profiling the distribution of electrons exciting the isochromat, and the second influencing the transmission of the excited radiation to the detector. These two functions build the total apparatus function of the BIS spectrometer. Few years ago, when analyzing deconvolution problems of the BIS spectra, we introduced a simple model of the apparatus function [1]. This model and some of its consequences will be developed and discussed below.

\section{Essentials of the BIS principle and equipment}

The typical instrumental arrangement for measurements of high energetic $\mathrm{X}$-ray bremsstrahlung isochromats is shown in Fig. 1. The details of the demountable X-ray tube are depicted in Fig. 2. The measured sample is placed on the anticathode of the $\mathrm{X}$-ray tube $(A)$. The electrons are emitted from an electron gun, which is usually a hot filament cathode $(K)$ with focusing system. They are accelerated to the kinetic energy $E_{\text {kin }}$ which is variable in the isochromat spec-

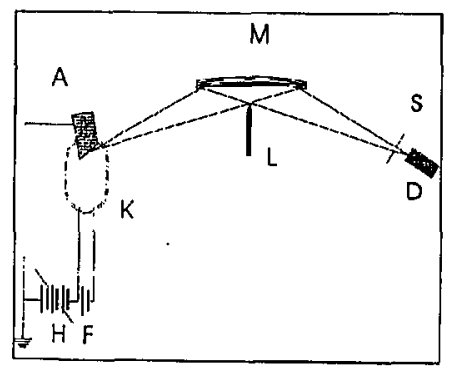

Fig. 1. Diagram of the BIS spectrometer. $A$ - anode with sample, $K-$ cathode, $M$ - monochromator of the Johamn type, $L-$ knife, $S$ - detector slit, $D$ - detector, $H$ - high voltage supply, $F$ - cathode supply. 


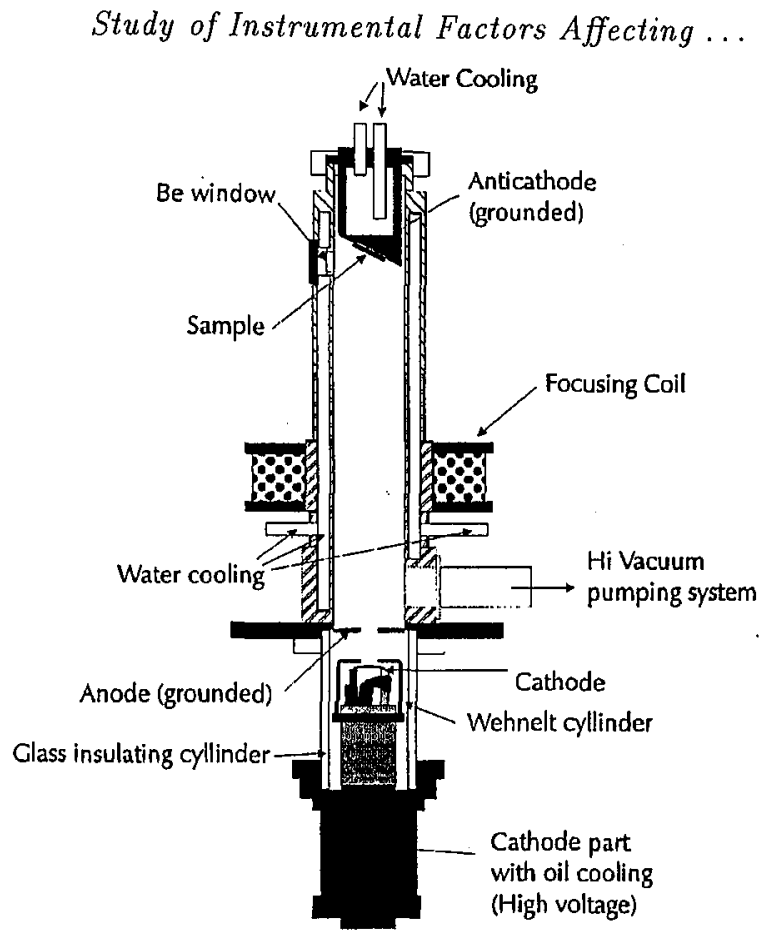

Fig. 2. General scheme of demountable X-ray tube used for the BIS measurements.

troscopy. Deceleration process of the electrons in the sample target produces the electromagnetic quanta. A part of these quanta, of the energy around the $E_{0}$ chosen by monochromator $(M)$, are registered in the detector $(D)$ and the intensity in function of kinetic energy of the excited electrons creates an isochromat spectrum. The monochromator position is fixed during the measurement.

A Johann type monochromator was used in this work with cylindrically bent $\mathrm{Si}(220)$ working in 1st order of reflection. Its active part was limited by the knife $(L)$. The parameters of the monochromator are collected in Table. The electron beam was focused on the sample by coaxial external coil. As a detector a proportional counter was used. One should notice that it is important to apply the lowest order of reflection in such types of apparatus. Applying the second or higher orders of reflections makes the monochromator pass also at the same angle quanta of twice lower energy. The counter registers some part of these quanta as "second harmonic". It was proved by us on other quartz (1010) monochromator that it leads to at least two effects: a significant increase in the background which is evident at the beginning of the isochromat and a slight lowering of the intensity at the higher energies of the spectra connected with the increase in the counter dead time (the quanta of the lower order can even "saturate" the counter in some cases).

The anode and anticathode of the X-ray tube were grounded and the high negative voltage was applied to the cathode. The cathode itself was supplied by separate power supply, and there was about $1 \mathrm{~V}$ potential difference between the 
TABLE

Values of basic parameters for the crystal used as monochromator in the BIS spectrometer.

\begin{tabular}{c|l|l}
\hline \hline 1 & Crystal & $\begin{array}{l}\text { Si }(220) \\
\text { cylindrically bent }\end{array}$ \\
\hline 2 & Order of reflection & 1 \\
\hline 3 & $K_{\alpha_{1}}$ Cr Bragg angle & 36.6004 \\
\hline 4 & $K_{\alpha_{2}}$ Cr Bragg angle & 36.6730 \\
\hline 5 & Radius of curvature & $1000 \mathrm{~mm}$ \\
\hline 6 & Dispersion $\left(K_{\alpha_{1}}\right.$ Cr) & $3.0825 \times 10^{-3} \AA / \mathrm{mm}$ \\
\hline 7 & $\begin{array}{l}\text { Geometrical linewidth } \\
\text { (knife }=2 \text { mm) }\end{array}$ & $0.036 \mathrm{eV}$ \\
\hline 8 & $\begin{array}{l}\text { Vertical divergence } \\
\text { (crystal height }=20 \mathrm{~mm})\end{array}$ & $0.49 \mathrm{eV}$ \\
\hline 9 & $\begin{array}{l}\text { Slit broadening } \\
(s=0.9 \mathrm{for} h=7 \mathrm{~mm})\end{array}$ & $0.72 \mathrm{eV}$ \\
\hline 10 & $F_{\mathrm{s}}$ FWHM calculated & $1.24 \mathrm{eV}$ \\
\hline 11 & $F_{\mathrm{s}}$ FWHM experimental & $1.1 \mathrm{eV}$
\end{tabular}

ends of the cathode. The influence of this voltage on the apparatus function as well as some changes in construction allowing to lower this influence will be discussed later.

\section{Apparatus function of the BI spectrometer}

If both the electron energy distribution and spectrometer window were infinitely narrow, the spectra would be free of most of the instrumental influences. Any smearing in such spectra would be due to physical processes, like lifetime broadening or many-body effects only. It would be possible to make a simple comparison with the uDOS calculations. In the realistic case however, both electron energy distribution function, $F_{\mathrm{e}}$, and spectrometer window, $F_{\mathrm{s}}$, are of finite width giving thus contribution to broadening of the measured real spectrum. It is widely accepted that the true isochromat, $I_{2}$, can be considered as convolution of the ideal isochromat, $I_{1}$, with the two functions $F_{\mathrm{e}}$ and $F_{\mathrm{s}}$ :

$$
I_{2}(E)=I_{1}(E) * F_{\mathrm{e}} * F_{\mathrm{s}},
$$

where $*$ denotes convolution operation. The total apparatus function is conventionally defined as

$$
F_{\mathrm{t}}=F_{\mathrm{e}} * F_{\mathrm{s}} .
$$

To obtain the "pure physical" isochromat $I_{1}$ one should deconvolute Eq. (1):

$$
I_{1}=I_{2} / F_{\mathrm{t}}
$$

here / denotes the deconvolution process. 


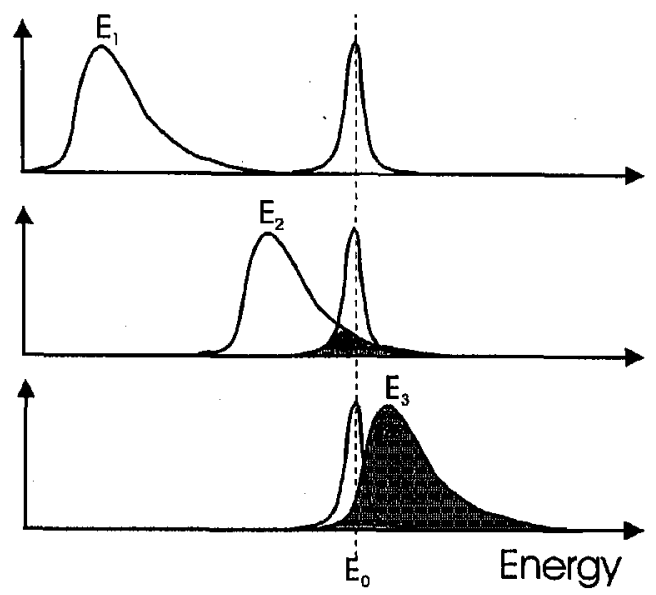

Fig. 3. Relations between two components of the total apparatus function in various stages of spectrum measurement. See text for explanation.

To do this in a simple way (e.g. with Fourier deconvolution or an iterative method) we must be sure that that the apparatus function is independent of excitation energy. It is usually supposed to be the case. To test whether it is really true, let us assume, for clarity, $F_{\mathrm{e}}$ to be much broader with respect to $F_{\mathrm{s}}$ (Fig. 3 ). If the excitation energy is much lower than the quantum energy $E_{0}$. defined by the monochromator setting (Fig. 3, upper) no part of the electron distribution $F_{\mathrm{e}}$ gives rise to the isochromat intensity. This generates the background region of the isochromat spectra. If the excitation energy is close to the $E_{0}$ only part of $F_{\mathrm{e}}$ of energies higher than $E_{0}$ is involved in the creation of isochromat (Fig. 3, middle). The last case (Fig. 3, bottom) responds to the situation when the excitation energy is sufficiently higher than the $E_{0}$ enabling thus all electrons to contribute to the isochromat spectrum. Only in the latter case Eq. (2) describing the total apparatus function is valid. When the excitation energy is close to or less than $E_{0}, F_{\mathrm{t}}$ is no longer independent of energy.

Typical proportions of $F_{\mathrm{e}}$ and $F_{\mathrm{s}}$ depend strongly on the details of spectrometer construction. In the spectrometer used in this study, the Lorentzian of FWHM equal to $1-2 \mathrm{eV}$ can represent $F_{\mathrm{s}}$. The values obtained experimentally agree with the calculations based on the Johann spectrometer theory (see Table). The FWHM of the function standing for the asymmetric electron energy distribution from hot cathode of the temperature of $2300 \mathrm{~K}$ is of the order of $0.5 \mathrm{eV}$. In unsophisticated electron guns $F_{\mathrm{e}}$ can be even broader due to the Boersch effects strongly dependent on the construction of the gun and the X-ray tube $[2,3]$. Long electron trajectories in the X-ray tube (more than $30 \mathrm{~cm}$ ) and high currents applied (of the order of 10-15 $\mathrm{mA}$ ) are among the most important reasons of the Boersch broadening. It was experimentally measured by us that about $80 \%$ of the total X-ray tube current reached the anticathode. During the EXBIFS measurements one should also take into account a systematic change in the electron distribution with increase in the voltage as a result of defocusing of the external coil, which is 
usually supplied with the constant current. It can slightly decrease the isochromat intensity with the voltage increase.

To avoid the additional smearing of the electron distribution coming from the small voltage along the cathode wire (of about $1 \mathrm{~V}$ ) an electronic system was applied enabling to turn off the voltage from the anode with a frequency of $10-50 \mathrm{~Hz}$ and to count the spectra only in these short periods. Surprisingly, this system did not improve significantly the resolution of the apparatus. It can be explained by two reasons. The first is due to electron emission distribution from the cathode wire; it can be expected that only small part in the middle of the cathode takes effectively part in the electron emission (because of higher heat transport at the ends of cathode, the hottest, and the thinnest part should be expected in the middle). The second reason can be connected with the Boersch effects themselves. However, it is difficult to estimate which of these factors are more important here.

From the above considerations one can conclude that the region of variable apparatus function is at least of the order of sum of halfwidths of $F_{\mathrm{e}}$ and $F_{\mathrm{s}}$. In most of the isochromat spectrometers working in the region of X-rays of few $\mathrm{keV}$ quantum energy it is equal to $1.5 \mathrm{eV}$ or more near the threshold. In the spectrometers working with lower quantum energies this value can be smaller. This result should be taken into account when one wishes to compare precisely the measured isochromat spectra with some theoretical calculations (e.g. uDOS). It is also clear that deconvolution of the isochromats by means of methods requiring constant kernel functions is possible only with $F_{\mathrm{s}}$, but not with the total apparatus function. For correction of the measured isochromats for $F_{\mathrm{t}}$ more general methods based on the Fredholm equation should be employed. However, to do this it is necessary to know exact shape of the $F_{\mathrm{e}}$ distribution, which is experimentally often a hard task.

\section{Experimental methods of the apparatus function estimation}

Let us consider now the problem how to measure the apparatus function of isochromat spectrometer. Two methods are widely applied to estimate the function. The principle of the first of them, "isochromat edge test" is based on an assumption that there is a target with step-like uDOS close to the Fermi level. The most common metals used in such kind of tests are Al, Ag or Au. It is expected that the derivative of an isochromat edge of the sample represents the total apparatus function. However, the above treatment fails as precise method because, as discussed above, in the isochromat edge region $F_{\mathrm{t}}$ can vary strongly. This method leads to underestimation of the apparatus function FWHM. In Fig. 4 the BI spectrum of the pure Au (curve 2) with its derivative (curve 1) is shown. For comparison the uDOS for Au (curve 4) together with BI isochromat measured by Lang and Baer by the apparatus working at $1486.6 \mathrm{eV}$ ( $\mathrm{Al} K_{\alpha}$ line) with very good resolution (better than $0.5 \mathrm{eV}$ ) are shown. The total resolution of the spectrometer applied to our measurement results in value of about $4 \mathrm{eV}$.

The other method is "calibration spectrum measurements". Isochromat spectrometer is usually set to the wavelength of an emission line. Let us consider 


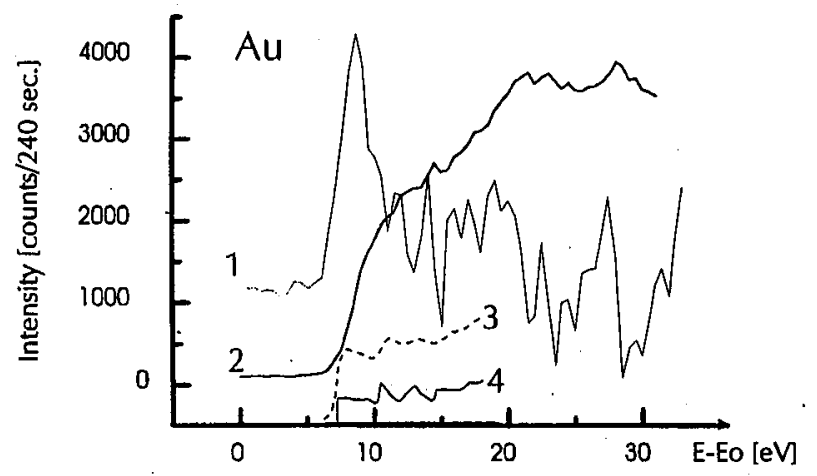

Fig. 4. BIS spectrum of pure Au (2), its derivative (1), uDOS for Au (4), BIS spectrum (3) measured by Lang and Baer [5].
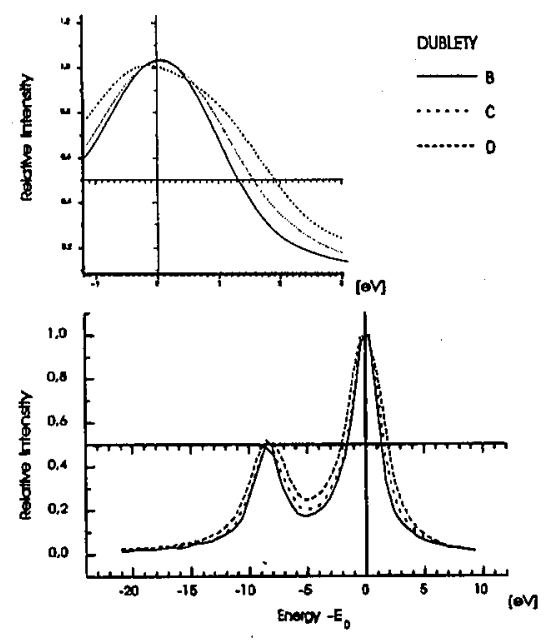

Fig. 5. Examples of the $\mathrm{Cr} K_{\alpha_{1} \alpha_{2}}$ doublet measurements for various knife settings (B $-1 \mathrm{~mm}, \mathrm{C}-2 \mathrm{~mm}, \mathrm{D}-3 \mathrm{~mm}$ ). Area near the main peaks enlarged in the upper picture.

Cr $K_{\alpha_{1}}$. Employing the $\mathrm{Cr}$ target and increasing suitably the $\mathrm{X}$-ray tube voltage one can measure the emission spectrum $\mathrm{Cr} K_{\alpha_{1} \alpha_{2}}$ by scanning with the detector slit (Fig. 5). From comparison of the measured spectrum with the natural linewidth one can estimate FWHM of $F_{\mathrm{s}}$. By deconvolution of one component (usually stronger $K_{\alpha_{1}}$ ) with a good measurement from double-crystal spectrometer it is possible even to find the shape of $F_{\mathrm{s}}$. One must notice that common deconvolution methods do not converge in case of multipeak kernel, hence a need of separation of the single peak in order to use it as a kernel. The above method estimates $F_{\mathrm{s}}$, but says nothing about $F_{\mathrm{e}}$. Examples of the $\mathrm{Cr} K_{\alpha_{1} \alpha_{2}}$ doublet measurements are shown in Fig. 5 for various knife settings (of 1,2 , and $3 \mathrm{~mm}$ ). For comparison, the halfwidth of the $K_{\alpha_{1}}$ peak measured by Tsutsumi with resolution 
better than $0.2 \mathrm{eV}$ was about $1.95 \mathrm{eV}$ [4]. Assuming that doublet components and $F_{s}$ are Lorentzians in shape we can estimate (using the upper part of Fig. 5) FWHM of $F_{\mathrm{s}}$ as $0.7,1.2$ and $1.8 \mathrm{eV}$ for the curves $B, C$ and $D$, respectively.

From the above it is evident that there is no direct method to measure or estimate $F_{\mathrm{t}}$. It is possible only to evaluate $F_{\mathrm{s}}$ from the calibration spectrum. To get information about total apparatus function, $F_{\mathrm{t}}$, it is necessary to measure the electron energy distribution at the target position. Unfortunately it was not possible in this study.

\section{Discussion and concluding remarks}

The problem of BIS spectra deconvolution has been discussed in paper [1]. There was shown how the results of the isochromat deconvolution vary with changes in shape and symmetry of the apparatus function. In order to avoid the "cut-off error" arising from large intensities of the high energetic end of isochromat, to the end of the original experimental data the mirror reflection of the same data is usually appended. It makes problem of the deconvolution error estimation difficult to calculate, and therefore, some results of such treatment are questionable.

It is clear that without knowledge of the exact shape of the apparatus function in case of correction by deconvolution it is difficult to avoid results which can be significantly influenced by artifacts coming from improper choice of the kernel function. On the other hand, deconvolution carefully applied to the isochromat raw data, can reveal interesting features, often invisible in the original spectrum. An example is shown in Fig. 6, where it was possible, after deconvolution, to compare the isochromat spectra measured with relatively bad resolution, with theoretical uDOS calculations. The deconvolution was calculated with Lorentzian $F_{\mathrm{s}}$ of $3 \mathrm{eV}$ FWHM. The solid line is applied to pure $\mathrm{Nb}$ and the dotted line to $\mathrm{Nb}_{3} \mathrm{Sn}$ spectra. The zero of the energy scale is related to the Fermi level and it was found after adjusting to the DOS calculations.

General features of the measured $\mathrm{Nb}$ and $\mathrm{Nb}_{3} \mathrm{Sn}$ isochromats seem to be well correlated to the appropriate unoccupied DOS calculations. In Fig. 6 it can be seen that the main isochromat maximum is for $\mathrm{Nb}_{3} \mathrm{Sn}$ sample broader and more "flat". Also its center is positioned at slightly lower energies comparing to the pure $\mathrm{Nb}$ spectrum. It reflects the DOS changes: the main DOS peak being doubled and broader in the case of $\mathrm{Nb}_{3} \mathrm{Sn}$; position of its "gravity center" is at lower energy comparing to that in $\mathrm{Nb}$ metal. In DOS calculations of $\mathrm{Nb}_{3} \mathrm{Sn}$, at higher energies, additional well pronounced peak appears. In the isochromat measurements it results in more shallow first minimum with characteristic fold near the minimum, which does not exist in pure $\mathrm{Nb}$ spectrum. Density of states close to the Fermi level is, according to calculations, much larger comparing to the main unoccupied DOS peaks in superconducting $\mathrm{Nb}_{3} \mathrm{Sn}$, than it is in $\mathrm{Nb}$ metal. The slope of isochromat low energetic rising part is significantly larger for $\mathrm{Nb}_{3} \mathrm{Sn}$ spectrum which reflects the mentioned above feature. However one should take into consideration that this DOS peak near the Fermi level although relatively high is very narrow and thus can be smeared with various factors. This can lead to slope observed in the isochromat. 


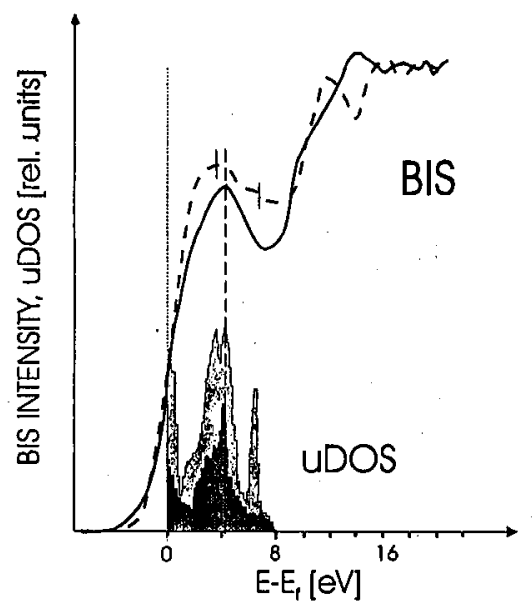

Fig. 6. Comparison of the isochromat spectra after deconvolution with Lorentzian $F_{\mathrm{s}}$ of $3 \mathrm{eV} \mathrm{FWHM}$ and DOS calculations. Dotted line: BIS of $\mathrm{Nb}_{3} \mathrm{Sn}$, solid line: BIS of pure $\mathrm{Nb}$, black diagram: uDOS of $\mathrm{Nb}$ metal calculated by K.M. Ho et al. [6], grey diagram: uDOS of $\mathrm{Nb}_{3} \mathrm{Sn}$ calculated by Jarlborg [7].

From the above it is evident that in the case presented, the initial structure of BI spectra measured for $\mathrm{Nb}$ and $\mathrm{Nb}_{3} \mathrm{Sn}$, which spreads out up to $10-12 \mathrm{eV}$ above the threshold, shows satisfactory correlations to the unoccupied parts of theoretical DOS calculations for these materials. Later measurements of $\mathrm{Nb}$ isochromat with resolution improved to $F_{\mathrm{s}} \mathrm{FWHM}$ of $1.2 \mathrm{eV}$ fairly agreed with the results described here.

In conclusion, it is noteworthy that correction of isochromat spectra by deconvolution cannot replace improvement of the spectrometer resolution if it is feasible. The uncertainty of the deconvolution process, its sensitivity to the kernel function shape and difficulties in estimation of an error of the method make the profits of the deconvolution often problematic when applied without sufficient experience.

It has been shown in this work that the total apparatus function of a BI spectrometer is not necessarily constant. The region of variable apparatus function is at least of the order of sum of halfwidths of $F_{\mathrm{e}}$ and $F_{\mathrm{s}}$. In most of the isochromat spectrometers working in the region of $X$-rays of few keV quantum energy it is $1.5 \mathrm{eV}$ or more near the threshold. In the spectrometers working with lower quantum energies this value can be smaller. The problem of the apparatus function in the BI spectroscopy and its influence on the spectra needs still more studies and experimental work to clarify its detailed properties and to analyze various methods of the spectra correction.

\section{Acknowledgments}

This work was partially sponsored by the State Committee for Scientific Research (Republic of Poland) (grant no. PB 0677/P3/93/04). 


\section{References}

[1] J. Pełka, Proc. 2nd Int. Seminar on X-ray and Electron Spectr., Madralin (Poland) 1989, Ed. J. Auleytner, K. Eawniczak-Jabłońska, Proc. Conferences in Physics, Polish Acad. Sci., Institute of Physics, Warsaw 1989, p. 171.

[2] H. Boersch, Z. Phys. 139, 115 (1954).

[3] B. Zimmermann, Adv. Electron. Phys. 29, 257 (1970).

[4] K. Tsutsumi, H. Nakamori, Proc. Int. Symp. X-Ray Spectra and Electronic Structure of Matter, Eds. A. Faessler, G. Wiech, Munich 1973, p. 100.

[5] J.K. Lang, Y. Baer, Rev. Sci. Instrum. 50, 221 (1979).

[6] K.M. Ho, S.G. Louie, J.R. Chelikowsky, M.L. Cohen, Phys. Rev. B 15, 1755 (1977).

[7] T. Jarlborg, P.O. Nilsson, J. Phys. C, Solid State Phys. 12, 265 (1979). 